\title{
LA ENERGÍA MINIHIDRÁULICA EN LOS PLANES DE FOMENTO DE LAS ENERGÍAS RENOVABLES EN ESPAÑA
}

\author{
Cayetano Espejo Marín \\ Ramón García Marín* \\ Universidad de Murcia \\ Ana Eulalia Aparicio Guerrero** \\ Universidad de Castilla-La Mancha
}

\section{RESUMEN}

La energía minihidráulica incluye las centrales con una potencia instalada de hasta 10 megavatios. Esta tecnología de producción energética se ha incluido en los Planes de Fomento de las Energías Renovables promovidos por los Gobiernos de España desde finales de los años noventa a la actualidad. En esta aportación se analizan los objetivos de potencia a instalar en cada Comunidad Autónoma durante el periodo de vigencia de cada Plan, las barreras con las que se encuentra el sector para el desarrollo de la energía minihidráulica y las actuaciones que se deberían llevar a cabo para la construcción de nuevas centrales. Desde 1998 a 2013 el balance ha sido muy positivo por el destacado incremento en el número de centrales (rehabilitadas y nuevas), en la potencia instalada y en la electricidad producida. Este hecho debe tenerse muy en cuenta porque esta energía es de origen renovable y sus instalaciones, debido a su reducido tamaño, tienen escaso impacto paisajístico y ambiental.

Palabras clave: Energía hidroeléctrica, central minihidráulica, energía renovable, Política Energética, España.

\footnotetext{
*cespejo@um.es, ramongm@um.es

**Ana.Aparicio@uclm.es
} 


\section{ABSTRACT}

The Small Hydropower in the Plans of promotion of renewable energies in Spain

The small hydropower includes plants with an installed capacity of up to 10 megawatts. This energy production technology has been included in the Plans of Development of Renewable Energies promoted by the governments of Spain since the late nineties of the last century to the present. In this research, the objectives proposed to install this kind of power in each region of Spain for the period of validity of each Energy Plan are analyzed, barriers in this sector for its development and the actions that should be carried out for the construction of new power plants. From 1998 to 2013, the balance has been very positive due the remarkable increase in the number of plants (new and rehabilitated), in installed capacity and electricity produced. This positive situation is of great interest because the small hydropower is of renewable origin, and its facilities, due to their small size, have a meager landscape and environmental impact.

Keywords: hydropower, small hydropower plant, renewable energy, energy policy, Spain.

La energía hidroeléctrica es aquella que se obtiene de aprovechar la energía potencial de una masa de agua situada en el cauce de un río para convertirla primero en energía mecánica y posteriormente en energía eléctrica. Por tanto, una central hidroeléctrica esta constituida por el conjunto de instalaciones y equipos necesarios para transformar la energía potencial de un curso de agua en energía eléctrica disponible. Existen dos tipologías básicas de aprovechamientos hidroeléctricos (Instituto para la Diversidad y Ahorro de la Energía, 2011):

- Centrales de agua fluyente: son los aprovechamientos que, mediante una obra de toma, captan una parte del caudal circulante por el rio y lo conducen hacia la central para ser turbinado y posteriormente restituido al rio. Este tipo de centrales se mueve en rangos de potencia bajos, normalmente inferiores a 5 megavatios (MW) y tienen una cuota del 75\% del mercado. En este tipo se incluyen también las centrales en canal de riego, que utilizan el desnivel del agua en los canales de riego para producir electricidad. El rango de potencia de las centrales utilizadas es de entre 1 y 5 MW y pueden suponer el 5\% del mercado en España.

- Centrales de pie de presa: son los aprovechamientos que, mediante la construcción de una presa o utilización de una existente con posibilidades de almacenar las aportaciones del rio, pueden regular los caudales a turbinar en el momento preciso. Estas centrales suelen tener unos niveles de potencia superiores a los $5 \mathrm{MW}$ y suponen aproximadamente el 20\% del mercado en España. Dentro de esta tipología, cabe destacar, 
por las perspectivas futuras que pueden tener, las centrales de bombeo o reversible, plantas que, además de funcionar como una central convencional generando energía (modo turbinación), tienen la capacidad de elevar el agua a un embalse o depósito consumiendo energía eléctrica (modo bombeo). Se pueden clasificar en dos tipos: las de bombeo puro, en las que el embalse superior es un gran depósito cuya única aportación de agua es la que se bombea del embalse inferior, y las de bombeo mixto, en las que el embalse superior tiene aportaciones naturales. En este caso, la Directiva 2009/28/CE de fomento del uso de energías procedentes de fuentes renovables, establece que la electricidad producida en unidades de acumulación por bombeo que utilizan agua que se ha bombeado aguas arriba no debe considerarse electricidad producida a partir de fuentes renovables (Instituto para la Diversidad y Ahorro de la Energía, 2011).

En los primeros años del desarrollo del sector eléctrico español, las centrales conocieron un gran auge y fueron incluso la base de buena parte de la electrificación de los núcleos rurales. Con el tiempo, la tendencia del desarrollo hidroeléctrico se centró en instalaciones de gran potencia y un gran número de minicentrales fueron abandonadas por su escasa rentabilidad. Sin embargo, dos criterios básicos de política energética, seguida tanto por los países de la Unión Europea, como por gran parte del resto de los países desarrollados, han generado un renovado interés por este tipo de instalaciones. Por un lado, el objetivo de disminuir la dependencia energética, reduciendo sobre todo el uso de combustibles importados para la producción de electricidad, aconseja la mayor utilización posible de los recursos energéticos nacionales, entre los cuales se encuentran estas centrales de pequeña potencia. Por otro lado, los objetivos de preservación del medio ambiente y de eficiencia energética impulsan la utilización de las nuevas fuentes energéticas renovables, entre las cuales $-\mathrm{y}$ junto a la solar, la eólica o la biomasa, fundamentalmente-, se ha acordado a nivel internacional tener en cuenta la energía hidráulica aprovechable en base a minicentrales (UNESA, 2003).

Las primeras centrales hidroeléctricas españolas se construyen a finales del siglo XIX. Buena parte de la fase inicial del desarrollo eléctrico español estuvo ligado a la expansión de este tipo de instalaciones, ya que en 1901 el 40\% de las centrales existentes eran hidráulicas. En esta etapa el desarrollo hidroeléctrico se encuentra con la enorme dificultad de que la electricidad se generaba en forma de corriente continua, por lo que no era posible su transporte a larga distancia. Por tal motivo, sólo podían aprovecharse aquellos recursos hidroeléctricos que se encontraban próximos a los centros de consumo. Este hecho determinó la localización de algunas industrias. La verdadera expansión de la 
industria hidroeléctrica en España se inicia en el siglo xx y llega hasta nuestros días (Espejo y García, 2010).

En España, el primer desarrollo eléctrico vino marcado por dos características que la diferencian de otros países: el preferente carácter hidráulico de su producción, lo que condicionó el tipo de oferta eléctrica disponible, y la importancia de la demanda urbana pues la debilidad del sector industrial determinó que, durante años, fuera el alumbrado y el transporte en las ciudades el principal consumidor de electricidad de nuestro país. Por ello, al principio, antes de la invención del transformador de alterna, fue la demanda urbana la que incitó la producción eléctrica con generadores de vapor situados en los mismos lugares de consumo, ya que las limitaciones de almacenamiento y transporte de la nueva energía impedían cualquier otra forma de producción. Además, ello se ajustaba al modelo clásico de la primera industrialización basado en la sustitución de la energía hidráulica preindustrial por el carbón y la máquina de vapor, con la excepción de algunas minicentrales hidráulicas en antiguos molinos (Arroyo, 2012).

A principios del siglo xx se produjo una intensa construcción de pequeñas centrales hidroeléctricas en América del Norte, Europa y Asia. En los años 1920 la energía generada en centrales hidroeléctricas constituía un $40 \%$ del total producido mundialmente por las centrales en conjunto. Después, durante un largo periodo (50 años) tuvo lugar una auténtica caída en la construcción de pequeñas centrales hidroeléctricas, para dar cabida a las grandes centrales que ofrecían un mayor rendimiento económico. En España, entre 1964 y 1978 de un total de 1.740 centrales con una potencia de $600 \mathrm{MW}$ se mantuvieron en explotación 735 con una potencia total de $500 \mathrm{MW}$, lo que indica que se abandonaron las 1.000 centrales de menor potencia. (Santos, 2003).

Para estimular la construcción y rehabilitación de pequeñas centrales se han adoptado medidas que han favorecido la implantación de nuevas centrales y la rehabilitación, cuando ha sido posible, de otras abandonadas. En 1980 se creó la Comisión de Pequeñas Centrales Hidroeléctricas, que auspició la evolución del potencial hidroeléctrico aprovechable mediante pequeñas centrales hidroeléctricas. Los incentivos ofrecidos por las administraciones públicas se han ido legislando desde la Ley 82/1980 de Conservación de la Energía, en la que se establecen los beneficios a los que se pueden acoger con el fin de potenciar la construcción, ampliación o adaptación de las instalaciones de producción hidroeléctrica con potencias de hasta 5 MW. El Real Decreto 2366/1994 sobre producción de energía eléctrica por instalaciones hidráulicas, de cogeneración y otras abastecidas por recursos o fuentes renovables, en su artículo 2 indica que se pueden beneficiar de su régimen económico las centrales hidroeléctricas que se instalen o amplíen su potencia, siempre que no sea superior a los 10 MW. Esta capacidad es la que define como pequeños aprovechamientos a estas 
instalaciones en España, Portugal, Irlanda, Grecia y Bélgica (European Small Hydropower Association, 2006), y por tanto el tamaño que consideramos para las centrales minihidráulicas objeto de este estudio.

El resurgimiento de la energía minihidráulica en España se ha debido a la existencia de un marco legislativo de apoyo tarifario para los productores de electricidad con fuentes de energías renovables. La Ley 54/1997 del Sector Eléctrico establece un Régimen Especial para aquellas instalaciones que utilizan fuentes de Energía Renovables con una potencia instalada inferior a los $50 \mathrm{MW}$. Además, la Ley reconoce los beneficios ambientales de estas fuentes mediante la percepción de una prima, permitiendo a las renovables entrar en competencia con las tecnologías convencionales, sobre las que los costes externos (sociales y medioambientales) generados no están repercutidos (Espejo, 2005).

Entre los años 1999 y 2011 los Gobiernos de España han elaborado tres planes para el fomento de las energías renovables. En diciembre de 1999 se aprueba el Plan de Fomento de las Energías Renovables en España 2000-2010, en agosto de 2005 el Plan de Energías Renovables 2005-2010, y en noviembre de 2011 el Plan de Energías Renovables 2011-2020. En esta aportación se analiza el sector minihidráulico en estos Planes, para conocer su evolución desde finales del siglo pasado, los objetivos de crecimiento, las barreras que frenan su expansión y las propuestas para su desarrollo.

\section{El PLAN DE FOMENTO DE LAS ENERGÍAS RENOVABLES EN EsPaÑa (2000-2010)}

El Consejo de Ministros del Gobierno del Partido Popular, en su reunión del 30 de diciembre de 1999, aprueba el Plan de Fomento de las Energías Renovables para el periodo 2000-2010 (PFER). En él se recogen los principales elementos y orientaciones que pueden considerarse relevantes en la articulación de una estrategia para que el crecimiento de cada una de las áreas de energías renovables pueda cubrir, en su conjunto, cuando menos el $12 \%$ del consumo de energía primaria en el año 2010.

El PFER se elabora como respuesta al compromiso que emana de la Ley 54/1997, del Sector Eléctrico, y que define el objetivo de desarrollo a alcanzar por las energías renovables. El diseño de las actuaciones de fomento se realiza en el marco legal en el que operan las instalaciones de generación eléctrica y térmica con fuentes renovables, sobre la base de que el apoyo a dichas fuentes es necesario dada su contribución a los principales objetivos de la política energética nacional: la diversificación de las fuentes primarias para garantizar la seguridad del suministro, la eficiencia en su utilización y el respeto al medio ambiente junto a un impacto favorable en el tejido industrial.

Dentro del área hidroeléctrica el apartado 6 se dedica a la previsión del mercado. En él se indica que el potencial hidroeléctrico de un país es la capacidad 
anual de producción de energía hidroeléctrica que dicho país posee, mientras que el potencial técnicamente explotable se deduce del anterior considerando las pérdidas.

La evaluación más reciente del potencial hidroeléctrico de España se realizó en 1980, y contiene una distribución por cuencas (cuadro 1). Si a la evaluación realizada en 1980, se le descuenta el que ya está desarrollado así como las centrales que se encuentran en ejecución y en trámite concesional, puede deducirse el potencial restante técnicamente desarrollable y pendiente de realizar.

\section{Cuadro 1}

Distribución por cuencas hidrográficas del potencial hidroeléctrico en España. GWh/año (1980)

\begin{tabular}{|c|c|c|c|c|c|c|}
\hline \multirow{2}{*}{$\begin{array}{c}\text { Cuenca } \\
\text { Hidrográfica }\end{array}$} & \multicolumn{3}{|c|}{$\begin{array}{c}\text { Potencial de } \\
\text { futura utilización }\end{array}$} & \multirow{2}{*}{ Total } & \multirow{2}{*}{$\begin{array}{c}\text { Total } \\
\text { potencial } \\
\text { técnicamente } \\
\text { desarrollable }\end{array}$} & \multirow{2}{*}{$\begin{array}{c}\text { Potencial } \\
\text { fluvial } \\
\text { bruto }\end{array}$} \\
\hline & $\begin{array}{c}\text { Potencial } \\
\text { diferenciado }\end{array}$ & $\begin{array}{l}\text { Aprovechamientos } \\
\text { medianos y grandes }\end{array}$ & $\begin{array}{c}\text { Aprov. } \\
\text { pequeños }\end{array}$ & & & \\
\hline Norte & 10.600 & 9.300 & 2.700 & 12.000 & 22.600 & 34.280 \\
\hline Duero & 6.700 & 4.200 & 600 & 4.800 & 11.500 & 29.400 \\
\hline Tajo & 3.900 & 4.200 & 600 & 4.800 & 8.700 & 16.540 \\
\hline Guadiana & 300 & 300 & 0 & 300 & 600 & 3.830 \\
\hline Guadalquivir & 400 & 500 & 300 & 800 & 1.200 & 10.410 \\
\hline Sur de España & 200 & 100 & 300 & 400 & 600 & 2.740 \\
\hline Segura & 100 & 600 & 100 & 700 & 800 & 2.090 \\
\hline Júcar & 1.200 & 1000 & 400 & 1.400 & 2.600 & 7.490 \\
\hline Ebro & 7.600 & 7000 & 1.400 & 8.400 & 16.000 & 40.060 \\
\hline $\begin{array}{l}\mathrm{P} \text { i r i n e o } \\
\text { Oriental }\end{array}$ & 600 & 100 & 300 & 400 & 1.000 & 3.520 \\
\hline Total cuencas & 31.600 & 27.300 & 6.700 & 34.000 & 65.600 & 150.360 \\
\hline
\end{tabular}

Fuente: Instituto para la Diversificación y Ahorro de la Energía. Plan de Fomento de las Energías Renovables.

A finales de 1999 el potencial hidroeléctrico pendiente de desarrollo es, para las centrales con una potencia igual o inferior de $10 \mathrm{MW}$, de 3.314 gigavatios hora $(\mathrm{GWh}) / a$ ño, con una potencia a instalar de $1.069 \mathrm{MW}$; y para las centrales entre 10 y $50 \mathrm{MW}$ de $15.383 \mathrm{GWh} /$ año, con una potencia a instalar de 7.691 MW.

Se considera que este potencial hidroeléctrico pendiente de desarrollar es muy difícil que llegue a aprovecharse a corto y medio plazo por diferentes motivos, entre otros, el hecho de que muchos de los aprovechamientos hidráulicos estén situados en tramos de ríos reservados para pesca o incluidos en Parques Nacionales.

Otras dificultades vienen dadas por la competencia en los usos del agua, la utilización exhaustiva de las aportaciones disponibles para riegos, la gran dificultad para la ocupación de tramos que serían afectados por las obras y 
embalses, y el que muchos aprovechamientos para ser utilizados, necesitan que el Estado construya la infraestructura necesaria (presas).

Los objetivos del presente PFER se fijan sobre la base de los aprovechamientos que están en fase de ejecución y en trámite concesional o proyecto por parte de la Administración. Se entiende por aprovechamientos en ejecución, aquéllos que han superado el trámite concesional, aun cuando no se haya iniciado aún la construcción del mismo. En proyecto, en cambio, están aquellos que aún no han obtenido la concesión y se encuentran en estudio por parte de las Confederaciones Hidrográficas (cuadro 2).

Los objetivos del área hidroeléctrica dentro del horizonte temporal del PFER, que abarca hasta el año 2010, después de analizar los recursos existentes así como la probabilidad de que sea posible su puesta en explotación en dicho periodo, estarán formados por los proyectos que se encuentran en ejecución y parte de las centrales que están tramitando la concesión. La distribución de estos objetivos de potencia a instalar para la minihidráulica quedan reflejados en el cuadro 3.

\section{Cuadro 2}

Distribución por Comunidades Autónomas de los aprovechamientos hidráulicos en ejecución y en proyecto $(\leq 10 \mathrm{MW})$

\begin{tabular}{|c|c|c|c|c|}
\hline \multirow[t]{2}{*}{ Comunidad Autónoma } & \multicolumn{2}{|c|}{$\begin{array}{c}\text { Centrales hidroeléctricas } \\
\text { en ejecución }\end{array}$} & \multicolumn{2}{|c|}{$\begin{array}{c}\text { Centrales hidroeléctricas } \\
\text { en proyecto }\end{array}$} \\
\hline & Número & $\begin{array}{c}\text { Potencia } \\
\text { MW }\end{array}$ & Número & $\begin{array}{c}\text { Potencia } \\
\text { MW }\end{array}$ \\
\hline Andalucía & 5 & 11,7 & 18 & 53,7 \\
\hline Aragón & 13 & 20,3 & 96 & 232,0 \\
\hline Asturias & 21 & 40,2 & 43 & 114,0 \\
\hline Baleares & 0 & 0 & 0 & 0 \\
\hline Canarias & 3 & 1,2 & 2 & 0,6 \\
\hline Cantabria & 9 & 12,8 & 3 & 3,2 \\
\hline Castilla y León & 6 & 8,5 & 108 & 211,7 \\
\hline Castilla-La Mancha & 55 & 86,1 & 36 & 55,5 \\
\hline Cataluña & 21 & 26,3 & 81 & 200,0 \\
\hline Extremadura & 2 & 5,9 & 21 & 57,6 \\
\hline Galicia & 25 & 64,4 & 21 & 32,2 \\
\hline Madrid & 4 & 2,4 & 1 & 0,07 \\
\hline Murcia & 1 & 1,3 & 0 & 0 \\
\hline Navarra & 12 & 17,8 & 53 & 53,7 \\
\hline País Vasco & 10 & 6,8 & 8 & 6,2 \\
\hline La Rioja & 4 & 3,1 & 10 & 8,6 \\
\hline C. Valenciana & 1 & 4,9 & 4 & 6,9 \\
\hline Total & 192 & 313,7 & 505 & $1.035,9$ \\
\hline
\end{tabular}

Fuente: Instituto para la Diversificación y Ahorro de la Energía. Plan de Fomento de las Energías Renovables. 


\section{Cuadro 3}

Objetivos del Plan de Fomento de Energías Renovables por Comunidades Autónomas. Centrales de $\leq 10$ MW (1999-2010)

\begin{tabular}{|l|c|c|}
\hline \multicolumn{1}{|c|}{ Comunidad Autónoma } & Potencia MW & Producción GWh/año \\
\hline Andalucía & 50 & 155 \\
\hline Aragón & 69 & 213,9 \\
\hline Asturias & 62 & 161,2 \\
\hline Baleares & 0 & 6,2 \\
\hline Canarias & 2 & 27,9 \\
\hline Cantabria & 9 & 709,9 \\
\hline Castilla y León & 229 & 167,4 \\
\hline Castilla-La Mancha & 54 & 195,3 \\
\hline Cataluña & 63 & 151,9 \\
\hline Extremadura & 49 & 139,5 \\
\hline Galicia & 45 & 6,2 \\
\hline Madrid & 2 & 12,4 \\
\hline Murcia & 4 & 176,7 \\
\hline Navarra & 57 & 27,9 \\
\hline País Vasco & 9 & 40,3 \\
\hline La Rioja & 13 & 40,3 \\
\hline C. Valenciana & 13 & 2.232 \\
\hline Total & 720 & \\
\hline & & \\
\hline
\end{tabular}

Fuente: Instituto para la Diversificación y Ahorro de la Energía. Plan de Fomento de las Energías Renovables.

Las previsiones realizadas por el Instituto para la Diversificación y Ahorro de la Energía (IDAE), redactor del Plan, para el año 2010 están basadas en las siguientes consideraciones, para la hidráulica de menos de $10 \mathrm{MW}$ :

- La potencia instalada que sería necesaria para obtener el potencial hidráulico desarrollable sería de $2.419 \mathrm{MW}$.

- La industria española posee capacidad suficiente para realizar a lo largo del horizonte del Plan de Fomento de las Energías Renovables 1.200 MW.

- La estimación realizada por las Comunidades Autónomas es de 966,7 MW.

- Las previsiones realizadas por la Asociación de Pequeños Productores y Autogeneradores de Electricidad con Fuentes de Energías Renovables 
(APPA) y la Fundación Agustín de Betancourt son respectivamente de 1.000 y $610 \mathrm{MW}$.

- Las previsiones del IDAE acordes con los recursos existentes y teniendo además en cuenta las expectativas de crecimiento a lo largo del periodo 1999-2010 se sitúan en el intervalo 600-800 MW.

\section{EL PLAN DE ENERGÍAS RENOVABLES EN ESPAÑA 2005-2010}

El Consejo de Ministros del Gobierno del Partido Socialista Obrero Español aprueba el 26 de agosto de 2005 el Plan de Energías Renovables 2005-2010 (PER 2005-2010).

Según el Gobierno, España mantiene desde hace tres lustros un notorio crecimiento del consumo de energía y de la intensidad energética. Nuestra creciente y excesiva dependencia energética exterior -cercana al $80 \%$ en los últimos años- y la necesidad de preservar el medio ambiente y asegurar un desarrollo sostenible, obligan al fomento de fórmulas eficaces para un uso eficiente de la energía y la utilización de fuentes limpias. Por tanto, el crecimiento sustancial de las fuentes renovables, junto a una importante mejora de la eficiencia energética, responde a motivos de estrategia económica, social y medioambiental, además de ser básico para cumplir los compromisos internacionales en materia de medio ambiente.

El PER 2005-2010 constituye la revisión del Plan de Fomento de las Energías Renovables en España 2000-2010 hasta entonces vigente. Con esta revisión, se pretende mantener el compromiso de cubrir con fuentes renovables al menos el $12 \%$ del consumo total de energía en 2010, objetivo de las políticas de fomento de las energías renovables en la Unión Europea desde la aprobación del Libro Blanco en 1997, y que en España fue establecido por la Ley del Sector Eléctrico y dio lugar al mencionado Plan de Fomento. Así mismo, este Plan 2005-2010 incorpora los otros dos objetivos indicativos para el año 2010$29,4 \%$ de generación eléctrica con renovables y $5,75 \%$ de biocarburantes en transporte-, adoptados con posterioridad al anterior plan.

El Balance del PFER durante el periodo 1999-2004 señala que, desde la aprobación de ese Plan hasta finales de 2004, el consumo global de energías renovables ha aumentado en España en dos millones setecientas mil toneladas equivalentes de petróleo (tep) anuales, un crecimiento significativo, aunque insuficiente para alcanzar los ambiciosos objetivos fijados. A finales de 2004, se había alcanzado un cumplimiento acumulado del $28,4 \%$ sobre el objetivo global de incremento de las fuentes renovables previsto para 2010.

Tres fuentes renovables han evolucionado hasta la fecha de forma satisfactoria: eólica, biocarburantes y biogás. La energía minihidráulica avanza más 
despacio de lo previsto. El PFER había fijado para el periodo 1999-2010 un objetivo de incremento en términos de energía primaria de 192 kilotoneladas equivalentes de petróleo (Ktep), y entre 1999 y 2004 este incremento ha sido de 64 Ktep. Menor crecimiento han tenido las centrales hidroeléctricas de 10 a 50 MW, con 7 Ktep frente a un objetivo de 60 Ktep.

Junto a la propia dinámica de seguimiento del PFER, que indica que las previsiones iniciales de crecimiento no se están cumpliendo, para el Gobierno de España existen razones adicionales que aconsejan la mencionada revisión.

En primer lugar, el consumo de energía primaria (y la intensidad energética) han crecido muy por encima de lo previsto en el escenario de ahorro al que se asociaron los objetivos del Plan de Fomento, en gran medida inducido por el importante incremento de la demanda eléctrica y del consumo de carburantes para el transporte. Y ello, por sí solo, obligaría a revisar al alza el crecimiento previsto de las energías renovables por el Plan de Fomento para alcanzar el 12\% en el año 2010. Ante esta situación, la Subdirección General de Planificación Energética del Ministerio de Industria, Turismo y Comercio, elabora unos nuevos escenarios de previsión energética que es necesario considerar en la vigente planificación, y en los que el consumo de energía primaria del año 2010 se sitúa claramente por encima del previsto en el Plan de Fomento de las Energías Renovables 2000-2010.

Igualmente, la revisión coincide con la elaboración de un «Plan de Acción 2005-2007», que plantea las líneas prioritarias de actuación para el lanzamiento de las medidas contempladas en la Estrategia de Ahorro y Eficiencia Energética en España 2004-2012 (E4) durante los próximos años.

También han de considerarse las ventajas de las energías renovables ante los nuevos compromisos de carácter medioambiental, muy especialmente los derivados del Plan Nacional de Asignación de Derechos de Emisión (PNA), aprobado por el Gobierno recientemente y, en general, los relativos al cumplimiento del Protocolo de Kyoto, en vigor desde el 16 de febrero de 2005.

Las fuentes de energías renovables se incorporan en el PNA 2005-2007 como instrumento fundamental para la reducción de las emisiones de $\mathrm{CO}_{2}$ en los sectores difusos, que no están cubiertos por la Directiva. El propio PNA ya anticipaba la necesidad de revisar los objetivos del Plan de Fomento de las Energías Renovables 2000-2010 en lo relativo al uso de biocarburantes para el transporte, con vistas a alcanzar los valores de consumo establecidos para los años 2005 y 2010 por la Directiva europea 2003/30/CE sobre biocarburantes, que se transpone a nuestra legislación a través del Real Decreto 1700/2003, de 15 de diciembre. 
Por todo ello, durante la evaluación realizada para la elaboración del PER 2005-2010, se trata de reforzar la coordinación en los tres ámbitos de planificación que se han mencionado -energías renovables, eficiencia energética y lucha contra el cambio climático-, buscando en todo momento la máxima eficacia y coherencia de objetivos e instrumentos y manteniendo en lo posible los compromisos anteriormente adquiridos.

El capítulo 3 del PER 2005-2010 incluye un exhaustivo análisis de las diferentes tecnologías consideradas, repasando en cada caso la situación existente en otros países del entorno europeo y evaluando los requerimientos necesarios para superar las principales barreras que frenan su desarrollo en España. Se formulan propuestas concretas de actuación y se definen los objetivos de cada área para el año 2010, que, en la mayoría de los casos, difieren de los objetivos que habían sido establecidos en el Plan de Fomento de las Energías Renovables vigente hasta entonces. Así mismo, se hace un repaso de la situación del sector industrial en España y se apuntan líneas de innovación tecnológica. En el área minihidráulica, la potencia instalada ha evolucionado favorablemente entre los años 1999 y 2004 (cuadro 4).

Por Comunidades Autónomas, los objetivos establecidos en el PER 20052010 más ambiciosos para el año 2010 se fijaron para Castilla y León, con 229 MW nuevos de potencia a instalar, seguida de lejos por Aragón con 69 MW y Cataluña con $63 \mathrm{MW}$, hasta totalizar un incremento nacional de $720 \mathrm{MW}$ de nueva potencia a instalar en centrales hidroeléctricas menores de $10 \mathrm{MW}$. El grado de cumplimiento del objetivo global es del $33 \%$ a finales de 2004, si bien, a nivel autonómico, cabe destacar el caso de Galicia, que ha superado ampliamente los objetivos planteados en el Plan de Fomento (cuadro 5).

\section{Cuadro 4}

Potencia instalada minihidráulica en el periodo de vigencia del Plan de Fomento de Energías Renovables 1999-2010 (MW)

\begin{tabular}{|c|c|c|c|c|c|c|c|}
\hline Hasta 1998 & 1999 & 2000 & 2001 & 2002 & 2003 & 2004 & Total \\
\hline $1.509,6$ & 35,8 & 42,9 & 41,8 & 36,3 & 37,3 & 45,1 & 1.749 \\
\hline
\end{tabular}

Fuente: Instituto para la Diversificación y Ahorro de la Energía. Plan de Energías Renovables en España 2005-2010. 


\section{Cuadro 5}

Objetivos por Comunidades Autónomas en el Plan de Fomento de las Energías Renovables 1999-2010 e incremento de potencia minihidráulica en el periodo de vigencia

\begin{tabular}{|l|c|c|}
\hline \multicolumn{1}{|c|}{ Comunidad Autónoma } & Objetivo de Potencia MW & Situación año 2004 MW \\
\hline Andalucía & 50 & 11 \\
\hline Aragón & 69 & 20,6 \\
\hline Asturias & 62 & 9,5 \\
\hline Baleares & 0 & 0 \\
\hline Canarias & 2 & 2 \\
\hline Cantabria & 9 & 38,5 \\
\hline Castilla y León & 229 & 10,3 \\
\hline Castilla-La Mancha & 54 & 21,4 \\
\hline Cataluña & 63 & 5,6 \\
\hline Extremadura & 49 & 80,6 \\
\hline Galicia & 45 & 0,2 \\
\hline Madrid & 2 & 1,3 \\
\hline Murcia & 4 & 20,7 \\
\hline Navarra & 57 & 8,8 \\
\hline País Vasco & 9 & 3,4 \\
\hline La Rioja & 13 & 5,1 \\
\hline C. Valenciana & 13 & 239 \\
\hline Total & 720 & \\
\hline
\end{tabular}

Fuente: Instituto para la Diversificación y Ahorro de la Energía. Plan de Energías Renovables en España 2005-2010.

Según el Gobierno de España, el mantenimiento de la energía hidroeléctrica se ha debido a la existencia de un marco legislativo de apoyo tarifario para los productores de electricidad con fuentes de energías renovables. La Ley 54/1997 del Sector Eléctrico establece un Régimen Especial para aquellas instalaciones que utilizan fuentes de Energía Renovables con una potencia instalada inferior a los $50 \mathrm{MW}$, de manera que éstas no están obligadas a realizar ofertas al sistema, y al mismo tiempo, tienen garantizado el acceso a la red. El Real Decreto 436/2004 desarrolla la Ley del Sector Eléctrico, estableciendo el esquema legal y económico para el Régimen Especial, con el objeto de consolidar el marco regulador, y tratar de conferir mayor estabilidad y previsibilidad al sistema, mediante el establecimiento de un régimen económico duradero basado en una metodología de cálculo de retribución conocida. 
Como la normativa administrativa y técnica de funcionamiento y conexión a las redes eléctricas de centrales hidroeléctricas (Orden Ministerial de 5 de septiembre de 1985), se ha quedado desfasada fundamentalmente en los límites de potencia de las centrales a conectar a red, fijada actualmente hasta 5.000 kilovoltioamperios (kVA). En este caso, sí se está realizando la revisión de esta normativa y existe ya un borrador de Real Decreto sobre acceso a la red y condiciones de operación de instalaciones de producción de energía eléctrica de potencia igual o inferior a $50 \mathrm{MW}$.

La normativa básica en materia de aguas viene recogida en el texto refundido de la Ley de Aguas, aprobado por Real Decreto Legislativo 1/2001 de 20 de julio. Es de destacar por su importancia la Directiva 2000/60/CE del Parlamento Europeo y del Consejo de 23 de octubre, por el que se establece un marco comunitario de actuación en el ámbito de la política de aguas y cuya transposición supondrá modificaciones en la gestión del agua.

La tramitación administrativa para el otorgamiento de una concesión aparece desarrollada en el Reglamento de Dominio Público Hidráulico aprobado por Real Decreto 849/1986 de 11 de abril, modificado por el Real Decreto 606/2003. Además, existe un procedimiento de tramitación de concesiones y autorizaciones administrativas para la instalación, ampliación o adaptación de aprovechamientos hidroeléctricos con potencia nominal no superior a 5.000 kVA (Real Decreto 916/1985), lo que suponía una tramitación conjunta por los Organismos de cuenca y el Órgano competente en materia energética.

Por estos aspectos, considera el Gobierno que el sector hidroeléctrico no alcanza un mayor auge ya que la consecución de las necesarias concesiones de agua para la ejecución de un aprovechamiento hidroeléctrico representa su principal barrera, debido al largo y complicado procedimiento administrativo. Este proceso se encuentra, en muchos casos, estancado por la existencia de algún informe negativo o contrario al otorgamiento de organismos competentes en la materia, y que está ligado al otro tipo de barreras con las que cuenta la energía hidroeléctrica, como son las de tipo social y medioambiental, que frenan el mayor desarrollo del sector.

Respecto a la normativa medioambiental, con la entrada en vigor de la Ley 6/2001 de modificación del Real Decreto legislativo 1302/1986 de Evaluación de Impacto Ambiental, todos los proyectos de centrales hidroeléctricas incluidas en el Régimen Especial (menores de $50 \mathrm{MW}$ ) deben realizar la consulta al órgano ambiental de si deben o no someterse a una evaluación de impacto ambiental en la forma prevista en esta disposición.

Los aprovechamientos hidroeléctricos pertenecientes a las Comunidades Autónomas de Valencia, Castilla y León, Extremadura, Madrid, Murcia y País Vasco, deben someterse preceptivamente a dicho procedimiento, sea cual sea su potencia, de acuerdo con su respectiva norma autonómica. 
Para el establecimiento de los objetivos hidroeléctricos del PER 2005-2010 se tienen en cuenta los siguientes factores: la existencia de potencial hidroeléctrico pendiente de desarrollar en España, viable técnica y medioambientalmente; la disponibilidad de normativa favorable en cuanto al régimen económico de la energía hidroeléctrica, que permitirá incrementar la confianza y el interés de los promotores, para lograr un mayor desarrollo hidroeléctrico; las ventajas de contar con un sector industrial maduro; y también la existencia de tecnología y capacidad de fabricación nacional.

El potencial hidroeléctrico a desarrollar dentro del período 2005-2010 se fija sobre la base de los aprovechamientos hidroeléctricos que se encuentran en fase de ejecución y en trámite concesional o proyecto por parte de la Administración.

En el cuadro 6 se muestra la distribución por Comunidades Autónomas de la potencia a instalar de los aprovechamientos hidroeléctricos en ejecución y en proyecto para el área minihidráulica.

\section{Cuadro 6}

Distribución por Comunidades Autónomas de la potencia a instalar en minicentrales hidroeléctricas de aprovechamientos en ejecución y en proyecto. 2004

\begin{tabular}{|l|c|c|}
\hline \multicolumn{1}{|c|}{ Comunidad Autónoma } & Centrales en ejecución MW & Centrales en proyecto MW \\
\hline Andalucía & 10 & 57 \\
\hline Aragón & 8 & 42 \\
\hline Asturias & 11 & 75 \\
\hline Baleares & 0 & 2 \\
\hline Canarias & 1 & 0 \\
\hline Cantabria & 0 & 14 \\
\hline Castilla y León & 75 & 159 \\
\hline Castilla-La Mancha & 44 & 22 \\
\hline Cataluña & 24 & 107 \\
\hline Extremadura & 0 & 24 \\
\hline Galicia & 133 & 86 \\
\hline Madrid & 2 & 0 \\
\hline Murcia & 2 & 0 \\
\hline Navarra & 2 & 37 \\
\hline País Vasco & 2 & 5 \\
\hline La Rioja & 2 & 9 \\
\hline C. Valenciana & 9 & 647 \\
\hline Total & 323 & 8 \\
\hline
\end{tabular}

Fuente: Instituto para la Diversificación y Ahorro de la Energía. Plan de Energías Renovables en España 2005-2010. 
Una vez detectados los proyectos existentes en fase de ejecución y en proyecto, se analiza la probabilidad de su posible puesta en explotación dentro del período de vigencia del PER 2005-2010, descartando aquellos que presentan dificultades en su realización y que no se van a poder aprovechar a corto y medio plazo.

En el cuadro 7 se muestra, a modo indicativo, el desglose de los objetivos planteados para el año 2010 en el área minihidráulica en cada una de las Comunidades Autónomas, estableciendo en su conjunto un objetivo de potencia hidroeléctrica incremental de $450 \mathrm{MW}$ en el periodo 2005-2010, con lo que se lograría una potencia global de 2.199 MW en instalaciones minihidráulicas. Se espera que uno de los mayores incrementos de potencia minihidráulica instalada en el período 2005-2010 se produzca en Galicia, motivado fundamentalmente

\section{Cuadro 7}

Distribución indicativa por Comunidades Autónomas del objetivo de incremento de potencia al 2010 en el área minihidráulica

\begin{tabular}{|l|c|c|c|}
\hline $\begin{array}{c}\text { Comunidad } \\
\text { Autónoma }\end{array}$ & $\begin{array}{c}\text { Situación } \\
\text { Año 2004 } \\
\text { MW }\end{array}$ & $\begin{array}{c}\text { Incremento } \\
\mathbf{2 0 0 5 - 2 0 1 0} \\
\text { MW }\end{array}$ & $\begin{array}{c}\text { Potencia } \\
\mathbf{2 0 1 0} \\
\text { MW }\end{array}$ \\
\hline Andalucía & 198 & 30 & 228 \\
\hline Aragón & 194 & 40 & 234 \\
\hline Asturias & 90 & 10 & 100 \\
\hline Baleares & 0 & 0 & 0 \\
\hline Canarias & 1 & 1 & 2 \\
\hline Cantabria & 54 & 5 & 59 \\
\hline Castilla y León & 264 & 90 & 145 \\
\hline Castilla-La Mancha & 105 & 40 & 282 \\
\hline Cataluña & 232 & 50 & 32 \\
\hline Extremadura & 25 & 7 & 417 \\
\hline Galicia & 215 & 102 & 22 \\
\hline Madrid & 46 & 3 & 195 \\
\hline Murcia & 18 & 4 & 66 \\
\hline Navarra & 161 & 34 & 56 \\
\hline País Vasco & 55 & 11 & 58 \\
\hline La Rioja & 46 & 10 & 2.199 \\
\hline C. Valenciana & 45 & 13 & \\
\hline Total & 1.749 & 450 & 29 \\
\hline
\end{tabular}

Fuente: Instituto para la Diversificación y Ahorro de la Energía. Plan de Energías Renovables en España 2005-2010. 
por la evolución tan favorable experimentada en los cinco años de vigencia del Plan de Fomento (a finales de 2004, el incremento de potencia instalada en Galicia fue de 80,5 MW frente a los $45 \mathrm{MW}$ previstos) y también debido al gran número de proyectos otorgados pendientes de construcción. Por otro lado, se espera que las Comunidades Autónomas de Castilla y León, Cataluña y Aragón mantengan su tendencia de implantación de minicentrales hidroeléctricas, puesto que son las Comunidades que cuentan con mayores recursos hidroeléctricos pendientes de desarrollar.

La evolución anual prevista de la nueva potencia a instalar y de la producción eléctrica de origen minihidráulico con las nuevas instalaciones a poner en marcha durante la vigencia del Plan quedan recogidas en el cuadro 8.

\section{Cuadro 8}

Evolución anual prevista de la potencia a instalar y de la producción eléctrica en el área minihidráulica. 2005-2010

\begin{tabular}{|l|c|c|c|c|c|c|c|}
\hline & 2005 & 2006 & 2007 & 2008 & 2009 & 2010 & Total 2005-2010 \\
\hline $\begin{array}{l}\text { Incremento anual de } \\
\text { potencia MW }\end{array}$ & 70 & 70 & 70 & 80 & 80 & 80 & 450 \\
\hline $\begin{array}{l}\text { Producción anual } \\
\text { GWh }\end{array}$ & 109 & 326 & 543 & 775 & 1.023 & 1.271 & 4.046 \\
\hline
\end{tabular}

Fuente: Instituto para la Diversificación y Ahorro de la Energía. Plan de Energías Renovables en España 2005-2010.

En enero de 2011 la Agencia Estatal de Evaluación de las Políticas Públicas y la Calidad de los Servicios publica el documento Evaluación del Plan Español de Energías Renovables 2005-2010. En el cuadro 9 se expone el seguimiento de la potencia instalada y de la producción eléctrica.

Para los redactores del documento, el déficit de cumplimiento de los objetivos observado se debe, según los responsables del PER 2005-2010, a la dificultad de carácter administrativo para el procedimiento de concesiones. También se reconoce que pudieran haberse establecido unos objetivos en exceso ambiciosos para una fuente de energía de la que no existen previsiones razonables de aumento de potencia (aunque no se dispone de estudio alguno que evalúe la capacidad de aprovechamiento hidroeléctrico de las cuencas hidrográficas), ya que no hay mucho espacio disponible, y únicamente se trataría, en la mayoría de los casos, de mejorar la eficiencia de las instalaciones.

En el caso de la energía minihidráulica llegan a la conclusión que los agentes sociales manifiestan puntos de vista diferentes sobre distintos aspectos. Así, sobre la voluntad política para la aplicación de las medidas que lleven a la consecución de los objetivos, desde el ámbito empresarial se asegura que no 


\section{Cuadro 9}

Potencia instalada (MW) y producción eléctrica (GWh) en minicentrales hidroeléctricas. 2005-2010

\begin{tabular}{|l|l|c|c|c|c|c|c|c|}
\hline \multicolumn{2}{|c|}{$\begin{array}{c}\text { Objetivo } \\
\mathbf{2 0 1 0}\end{array}$} & 2005 & 2006 & 2007 & 2008 & 2009 & 2010 & $\begin{array}{c}\text { Acumulado } \\
2005-2010\end{array}$ \\
\hline \multirow{2}{*}{$\begin{array}{l}\text { Potencia } \\
\text { MW } \\
2.199\end{array}$} & $\begin{array}{l}\text { Previsiones } \\
\text { PER }\end{array}$ & 70 & 70 & 70 & 80 & 80 & 80 & 450 \\
\cline { 2 - 9 } & Real & 51,1 & 30,6 & 31,2 & 28,6 & 25,1 & 6,0 & 173 \\
\cline { 2 - 9 } & \% objetivo & $-27,0$ & $-56,3$ & $-55,4$ & 64,3 & 68,6 & $-92,5$ & $-61,6$ \\
\hline \multirow{2}{*}{$\begin{array}{l}\text { Producción } \\
\text { GWh } \\
3.257\end{array}$} & $\begin{array}{l}\text { Previsiones } \\
\text { PER }\end{array}$ & 109 & 326 & 543 & 775 & 1.023 & 1.271 & - \\
\cline { 2 - 9 } & Real & 77,7 & 202,7 & 297,8 & 297,8 & 494,1 & 585,1 & - \\
\cline { 2 - 9 } & \% objetivo & $-28,7$ & $-37,8$ & $-45,2$ & $-50,7$ & $-21,7$ & $-54,0$ & - \\
\hline
\end{tabular}

Fuente: Agencia Estatal de Evaluación de las Políticas Públicas y la Calidad de los Servicios.

existe apoyo político, pese al reducido impacto ecológico de la centrales, mientras que las organizaciones ecologistas señalan dimensiones medioambientales sensibles, cuando no negativas, de ciertos proyectos planteados, y se percibe, en este mismo sentido, un cierto rechazo social a estas instalaciones, lo cual deriva en una mayor dificultad de encontrar emplazamientos adecuados, y en la menor rentabilidad diferencial en relación con otras inversiones en el terreno de las energías renovables. Por último, se acusa a las grandes empresas eléctricas de obtener concesiones para instalaciones de minihidráulica que no utilizan, perjudicando así a los pequeños productores (Agencia de Evaluación y Calidad, 2011).

EL PLAN DE ENERGÍAS RENOVABLES 2011-2020

El Consejo de Ministros del Gobierno del Partido Socialista Obrero Español, en su reunión de 11 de noviembre de 2011, aprueba el Plan de Energías Renovables 2011-2020 (PER 2011-2020), estableciendo objetivos acordes con la Directiva 2009/28/CE del Parlamento Europeo y del Consejo, de 23 de abril de 2009, relativa al fomento del uso de energía procedente de fuentes renovables, y atendiendo a los mandatos del Real Decreto 661/2007, por el que se regula la actividad de producción de energía eléctrica en régimen especial y de la Ley 2/2011, de 4 de marzo, de Economía Sostenible.

Considera el Gobierno de España que el consumo acelerado de unos recursos energéticos finitos, el impacto ambiental asociado a la producción y uso de las energías tradicionales, la distribución de las reservas de energía, y los precios de las materias primas energéticas, confieren a las fuentes renovables de energía una importancia creciente en la política energética de la mayoría de los países 
desarrollados. La utilización de la energía procedente de recursos renovables constituye una parte muy importante en la estrategia de las políticas energética y medioambiental.

Para la Unión Europea, que tiene una fuerte dependencia energética, es sumamente importante aumentar progresivamente el grado de autoabastecimiento energético y solo será posible con la implantación progresiva de energías de futuro, sostenibles e inagotables, como las energías renovables. Para España, con una dependencia energética exterior aun mayor, resulta todavía más apremiante y estratégico avanzar con paso firme en este campo, mediante la transición y transformación del modelo energético hacia un nuevo «mix», en el que el papel de las energías renovables solo puede ser creciente a medio y largo plazo.

Por otra parte, la lucha contra el cambio climático es una prioridad política en materia de medio ambiente, tanto para la Unión Europea como para España. En este contexto, el uso de las energías renovables constituye una parte fundamental del paquete de propuestas necesarias para reducir las emisiones de gases de efecto invernadero y para cumplir con el Protocolo de Kioto y otros compromisos internacionales.

Con objeto de promover y facilitar el uso de los recursos renovables, desde mediados de los años ochenta, la Administración española ha publicado como herramienta principal sucesivos planes de energías renovables. Estos planes incluyen unos objetivos cuyo propósito es proporcionar seguridad a los inversores y promover el desarrollo de las tecnologías asociadas a estos recursos. La fijación de objetivos concretos en cada sector renovable exige un análisis específico de las barreras que se detectan en cada grupo y subgrupo, de manera que los Planes de Energías Renovables contemplen propuestas específicas para afrontarlas. Un posterior seguimiento de cada objetivo -en los balances anuales de los Planes- permite una correcta monitorización de cada sector y subsector, evaluando la necesidad de implantar actuaciones adicionales.

Finalizado el periodo de vigencia del PER 2005-2010 y atendiendo al mandato establecido en la legislación vigente procede la elaboración de un nuevo Plan, con el diseño de nuevos escenarios y la incorporación de objetivos acordes con la Directiva 2009/28/CE del Parlamento Europeo y del Consejo, de 23 de abril de 2009, relativa al fomento del uso de fuentes renovables, la cual establece objetivos mínimos vinculantes para el conjunto de la Unión Europea y para cada uno de los Estados miembros, y la necesidad de que cada Estado miembro elabore y notifique a la Comisión Europea, a más tardar el 30 de junio de 2010, un Plan de Acción Nacional de Energías Renovables (PANER) para el periodo 2011-2020, con vistas al cumplimiento de los objetivos vinculantes que fija la Directiva. Dicho PANER, tal y como prevé la Directiva, debía ajustarse al modelo de planes de acción nacionales adoptado por la Comisión Europea a través de la Decisión de la Comisión, de 30 de junio de 2009. El Estado Español, 
a través de la Secretaria de Estado de la Energía, presentó dicho plan dentro de los plazos establecidos por la Directiva.

La Directiva establece como objetivos generales, conseguir una cuota mínima del $20 \%$ de energía procedente de fuentes renovables en el consumo final bruto de energía de la Unión Europea, el mismo objetivo establecido para España, y una cuota mínima del 10\% de energía procedente de fuentes renovables en el consumo de energía en el sector del transporte en cada Estado miembro para el año 2020.

El PANER respondió a los requerimientos y metodología de la Directiva de energías renovables y se ajustó al modelo de planes de acción nacionales de energías renovables adoptado por la Comisión Europea.

E1 PER 2011-2020 incluye los elementos esenciales del PANER así como análisis adicionales no contemplados en el mismo y un detallado análisis sectorial que contiene, entre otros aspectos, las perspectivas de evolución tecnológica y la evolución esperada de costes. Además, tras la elaboración del PANER, y en el marco de una evolución muy negativa de la economía mundial y española, tuvieron lugar los trabajos de la Subcomisión de Análisis de la Estrategia Energética Española para los próximos 25 años, constituida en el seno de la Comisión de Industria, Turismo y Comercio del Congreso de la Diputados, que el 21 de diciembre de 2010 aprobó un documento con el apoyo de la mayoría de los grupos parlamentarios, en el que se recomendaba que la participación de las energías renovables fuera del $20,8 \%$ en el año 2020.

Ese es el objetivo global que se recoge en este Plan, que da respuesta, a su vez, al artículo 78 de la Ley 2/2011, de Economía Sostenible, que fija los mismos objetivos de la Directiva 2009/28/CE como los objetivos nacionales mínimos de energías renovables en 2020, estableciendo además que el Gobierno aprobará planes de energías renovables que hagan posible el cumplimiento de los objetivos fijados y que permitan la posibilidad efectiva de desarrollo de las energías renovables en todas las Comunidades Autónomas. La Directiva 2009/28/CE es parte del denominado Paquete Europeo de Energía y Cambio Climático, que establece las bases para que la UE logre sus objetivos para 2020: un 20\% de mejora de la eficiencia energética, una contribución de las energías renovables del $20 \%$ y una reducción de las emisiones de gases de efecto invernadero (GEI) del $20 \%$.

Sin embargo, teniendo en cuenta las conclusiones adoptadas por los Jefes de Estado y de Gobierno de la Unión Europea, podría materializarse un aumento en el objetivo de reducción de GEI hasta alcanzar el 30\% en 2020. En ese caso habrá que modificar los objetivos nacionales de reducción de estos gases y las políticas para conseguirlos, lo que podría suponer la revisión de los objetivos del PER 2011-2020. 
Dentro del periodo del Plan de Energías Renovables 2005-2010, a finales de 2010 se han instalado 173 nuevos MW en centrales hidroeléctricas de menos de $10 \mathrm{MW}$, por lo que el grado de cumplimiento en esta área ha sido del 38,4\% frente a los objetivos previstos al 2010 (cuadro 9).

Para la evolución prevista hasta el 2020, se ha tenido en cuenta la situación del sector tras la finalización del PER 2005-2010, el conocimiento de los proyectos en fase de tramitación administrativa, el potencial resultante de los estudios realizados para implantar aprovechamientos hidroeléctricos en infraestructuras de titularidad estatal (Convenio Instituto para la Diversificación y Ahorro de la Energía-Ministerio del Medio Ambiente, Medio Rural y Medio Marino de fecha 10/12/2007), así como la potencia que se viene instalando desde los últimos 10 años.

Para el PER 2011-2020 se ha revisado el escenario energético en el horizonte del año 2020, actualizando los datos a cierre del 2010, de forma que las energías renovables cubran en el año 2020 como mínimo el 20\% del consumo final bruto de energía en línea con los objetivos marcados por la Directiva 2009/28/CE. Por tanto, los objetivos globales del presente plan propuestos para el área hidroeléctrica, en términos de incremento de potencia instalada durante el periodo 20112020 , son los reflejados en el cuadro 10. El crecimiento anual previsto se estima siga la tendencia actual, si no hay ningún cambio en la legislación vigente desde noviembre de 2011, con incrementos de potencia media anuales de $25 \mathrm{MW}$, para llegar a 2020 con un aumento de $254 \mathrm{MW}, 24$ para las centrales de menos de 1 MW y 230 MW para las de 1 a 10 MW (cuadro 10).

\section{Cuadro 10}

Trayectoria de la capacidad a instalar en el sector hidroeléctrico al 2020 en el marco del Plan de Energías Renovables 2011-2020

\begin{tabular}{|c|c|c|c|c|c|c|c|c|c|c|c|}
\hline & 2010 & 2011 & 2012 & 2013 & 2014 & 2015 & 2016 & 2017 & 2018 & 2019 & 2020 \\
\hline \multicolumn{10}{|c|}{ POTENCIA MW } \\
\hline$<1$ & 242 & 244 & 247 & 249 & 251 & 243 & 256 & 259 & 262 & 265 & 268 \\
\hline $1-10$ & 1.680 & 1.687 & 1.695 & 1.703 & 1.731 & 1.764 & 1.796 & 1.828 & 1.855 & 1.882 & 1.917 \\
\hline TOTAL & 1.922 & 1.931 & 1.942 & 1.952 & 1.982 & 2.007 & 2.052 & 2.087 & 2.117 & 2.147 & 2.185 \\
\hline \multicolumn{10}{|c|}{ PRODUCCIÓN GWH } \\
\hline$<1$ & 802 & 804 & 748 & 791 & 779 & 772 & 839 & 821 & 803 & 887 & 843 \\
\hline $1-10$ & 5.432 & 5.118 & 6.197 & 5.075 & 5.007 & 4.982 & 4.857 & 5.058 & 5.249 & 5.441 & 5.744 \\
\hline TOTAL & 6.234 & 5.922 & 6.945 & 5.866 & 5.786 & 5.754 & 5.696 & 5.879 & 6.052 & 6.328 & 6.587 \\
\hline
\end{tabular}

Fuente: Instituto para la Diversificación y Ahorro de la Energía. Plan de Energías Renovables 2011-2020. 
Todos los documentos de los Planes Energéticos analizados cuentan con dos apartados: uno dedicado a las barreras al desarrollo del sector y otro con una serie de propuestas que sería necesario poner en marcha para facilitar un mayor ritmo de implantación de nuevas instalaciones. Estas propuestas comprenden las minicentrales hidroeléctricas y las que cuentan con una potencia entre $10 \mathrm{y}$ $50 \mathrm{MW}$, porque ambas, hasta la Ley del Sector Eléctrico de 2013, estaban en el grupo de instalaciones acogidas al régimen especial. Se relacionan las del PER 2011-2020.

\section{Barreras al desarrollo de la energía hidroeléctrica}

Para el desarrollo de nuevo potencial hidroeléctrico, las principales barreras detectadas son de recurso hidráulico y de tipo administrativo-medioambiental:

\section{Barreras de recurso hidráulico}

- Falta de conocimiento en detalle del potencial de recurso hidráulico por emplazamientos, que dificulta o ralentiza la realización de proyectos. Quedan todavía muchos emplazamientos en infraestructuras existentes (por ejemplo, en el Convenio entre el Ministerio del Medio Ambiente, Medio Rural y Marino de España y el Instituto para la Diversificación y Ahorro de la Energía, de diciembre de 2007 se identificaron 41 presas de titularidad estatal) o en zonas de mínima afección medioambiental, que pueden ser susceptibles de aprovechar hidroeléctricamente, de forma compatible con otros usos y desarrollables con criterios de sostenibilidad.

- Disminución de los recursos hídricos, por efecto del cambio climático, que afectará a la producción hidroeléctrica. Como ya se ha comentado en el apartado de evaluación del potencial, esta futura disminución de las aportaciones hidrológicas afectará sobre todo a las centrales hidroeléctricas de tipo fluyente, que no tienen capacidad de regulación, y se traducirá en una disminución en las horas equivalentes de funcionamiento.

\section{Barreras administrativas}

- Largas demoras para la obtención de las concesiones necesarias para el uso del agua, debido, entre otros motivos, a la dificultad para obtener la autorización medioambiental previa necesaria. Para el otorgamiento de la concesión de aguas para uso hidroeléctrico es condición necesaria que el proyecto haya obtenido la Declaración de Impacto Ambiental favorable por parte del Organismo ambiental. Fundamentalmente, dentro del proceso de tramitación concesional, largo y complicado, la mayor dificultad se encuentra en la obtención de la Declaración de Impacto 
Ambiental por parte del Organismo ambiental competente, ya que se vienen produciendo demoras de casi 2 años, lo que hace que el proceso global pueda llegar a durar hasta 10 años, desanimando a los inversores potenciales que preferirán otros proyectos más atractivos. También son comunes las dificultades en lograr conexiones a la red asequibles y no existen procedimientos rápidos para proyectos más pequeños.

- Oposición al otorgamiento de nuevas concesiones de agua de tipo fluyente por parte de determinados Organismos de Cuenca. Actualmente, la interpretación de la Directiva Marco del Agua (DMA) está provocando sensibilidades en algún Organismo de Cuenca contrarias a la explotación de aprovechamientos hidroeléctricos existentes y al futuro desarrollo de los mismos. El objetivo de esta Directiva es establecer un marco para la protección de las aguas continentales, costeras y subterráneas, que promueve un uso sostenible del agua, contribuye a paliar los efectos de inundaciones y sequias, mejora el medio acuático reduciendo vertidos y emisiones y previene todo deterioro adicional, mejorando el estado de los ecosistemas acuáticos y terrestres. En este sentido, la implantación de la DMA puede impedir el desarrollo del potencial fluyente disponible en cauces de ríos y la eliminación de minicentrales en desuso. Por otro lado, el establecimiento de los caudales de mantenimiento que se fijen en los instrumentos de planificación hidrológica puede afectar, en mayor o menor medida, a nuevos proyectos y a centrales en funcionamiento si los valores fijados son muy altos.

- Dificultades para la renovación del período de concesión de aguas en las centrales hidroeléctricas existentes. La no renovación de las concesiones puede derivar en abandono y sub-inversión de las plantas existentes durante los últimos años de explotación de las mismas.

- Procedimiento de tramitación concesional complejo, incluso para proyectos pequeños.

- Obstáculos para la obtención de las autorizaciones necesarias de los Organismos regionales y locales.

- Problemas para lograr conexiones a red asequibles.

\section{Actuaciones propuestas}

A continuación se indican una serie de propuestas que sería necesario poner en marcha para facilitar un mayor ritmo de implantación de nuevas instalaciones, de forma que se incrementasen los aprovechamientos hidroeléctricos de los recursos de forma compatible con la preservación de los valores ambientales y acordes con la planificación hidrológica y energética en el territorio nacional: 


\section{Propuestas económicas}

Se propone establecer un marco retributivo para la energía procedente de fuentes renovables, basado en el establecimiento de un suelo y techo remunerativo, los cuales garantizan una rentabilidad razonable a los promotores.

\section{Propuestas normativas}

- La simplificación de los procedimientos de autorización para aquellas instalaciones renovables eléctricas en las que la Agencia de Gestión de la Energía (AGE) sea competente, y para las instalaciones donde la AGE no sea competente, se propone la elaboración por parte del Ministerio de Industria, Turismo y Comercio de un catalogo de procedimientos y trámites a seguir para la implantación de estas instalaciones, de acuerdo con las directrices del artículo 84 de la Ley de Economía Sostenible.

- El desarrollo de una nueva reglamentación para la conexión a la red de media y baja tensión de instalaciones renovables de generación de energía eléctrica de baja potencia, hasta $100 \mathrm{Kw}$, fijando condiciones especiales técnicas y de tramitación para instalaciones de menos de 10 $\mathrm{Kw}$ asociadas a puntos de consumo. Esta medida se encuentra en elaboración y existe una Propuesta de Real Decreto.

- La modificación del Reglamento Eléctrico de Baja Tensión, mediante la creación de las Instrucciones Técnicas necesarias para cada tecnología de energías renovables que permitan regular las características técnicas que deben cumplir las instalaciones de pequeña potencia que se conecten en baja tensión.

- El establecimiento de un sistema de compensación de saldos de energía, denominado «balance neto», que permita a un consumidor que autoproduce parte de su consumo eléctrico, compatibilizando su curva de producción con su curva de demanda, mediante la cesión de excedentes puntuales al sistema y su posterior recuperación.

- El fomento de Empresas de Servicios Energéticos para un escenario futuro evolucionando hacia un esquema de generación distribuida de las energías renovables.

- El desarrollo de un marco normativo para promover la creación de nuevas centrales hidroeléctricas reversibles o la ampliación de existentes (presas, canales o depósitos), de forma compatible con la planificación hidrológica vigente y preservando los valores medioambientales. 
Propuestas específicas sectoriales

- El fomento de la rehabilitación de centrales hidroeléctricas. En España existe un gran potencial de rehabilitación y modernización de centrales hidroeléctricas, que ya han superado su vida útil y siguen funcionando muy por debajo de su nivel optimo con unos rendimientos muy bajos. Con la renovación de maquinaria e instalaciones se podrían conseguir mejoras en la producción eléctrica superiores a un $20 \%$, sin modificación de las condiciones concesionales, o bien adaptar esas condiciones a las posibles variaciones o disminución en los recursos hídricos, de forma que el nuevo equipamiento sea el optimo para las aportaciones hidrológicas existentes y recoja las condiciones previstas en los nuevos planes hidrológicos de cuenca, prestando especial atención a las políticas de implantación de caudales de mantenimiento. Se propone promover la rehabilitación, modernización y/o ampliación de centrales hidroeléctricas existentes, mediante la renovación de instalaciones deterioradas, sustitución de antiguos equipos por nuevos de alta eficiencia, implantación de nuevos sistemas de automatización y telegestión, conexión a la red eléctrica en el caso de centrales aisladas, etc., con el objetivo de mantener y/o aumentar la capacidad de producción de energía hidroeléctrica, de forma compatible con la preservación de los valores ambientales y acordes con la planificación hidrológica.

- El fomento de concursos para aprovechamientos hidroeléctricos en infraestructuras públicas existentes, llevados a cabo por parte de la Administración competente, la convocatoria de concursos públicos para el aprovechamiento hidroeléctrico de infraestructuras existentes de titularidad pública (presas, canales de riego, etc.) para otorgar concesiones de agua para producción eléctrica, de manera compatible con otros usos del agua y con los valores medioambientales.

- Nueva reglamentación para la tramitación de concesiones de agua o modificación de la existente, que resolviera distintos aspectos del marco legislativo actual, entre otros, los siguientes temas:

- Caducidad de concesiones.

- Procedimiento más ágil de forma que los tramites internos de los organismos de cuenca se simplificasen en un único informe conjunto de las distintas áreas.

- Posibilidad de modificación de las concesiones ya otorgadas, por la Autoridad que las concedió, sin necesidad de iniciar el trámite de competencia, cuando la modificación no produzca un incremento o decremento del caudal máximo ni de la potencia superior al 50\% de 
los valores concedidos y sea compatible con el Plan Hidrológico de la cuenca vigente.

- El impulso de proyectos de microcentrales hidroeléctricas en redes de abastecimiento u otras infraestructuras hidráulicas, mediante la instalación de micro turbinas hidroeléctricas en sistemas de abastecimiento a poblaciones u otros sistemas hidráulicos, que transformaran la energía en presión no utilizada en energía eléctrica, compatibilizando el uso principal de la infraestructura existente con la producción de energía.

\section{LOS RESULTADOS DE 15 AÑOS DE DESARROLLO DE LA ENERGÍA MINIHIDRÁULICA}

Desde 1998 a la actualidad las Políticas de Fomento de las Energías Renovables en España han permitido la recuperación y construcción de un abundante número de minicentrales, y con ello de la potencia instalada y de su contribución a la producción eléctrica nacional, a pesar de las dificultades con las que se ha encontrado este sector.

El seguimiento por parte de la Comisión Nacional de Energía, hoy denominada Comisión Nacional de los Mercados y la Competencia, del número de centrales, potencia instalada y producción de electricidad desde el año 1998 hasta la actualidad, permite disponer de información necesaria para conocer la evolución de la energía minihidráulica en nuestro país, y por tanto las consecuencias de las directrices de Política Energética, recogidas en las dos Leyes del Sector Eléctrico (1997 y 2013), los Planes de Fomento de las Energías Renovables (1999-2010, 2005-2010 y 2011-2020), así como de toda la legislación desarrollada a través de abundantes Reales Decretos. En el cuadro 11 se expone la evolución por Comunidades Autónomas para comparar la situación actual con la de finales del siglo pasado. En el conjunto de España, entre 1998 y 2013 se ha producido un incremento del $66 \%$ en el número de centrales, del $74 \%$ en la potencia instalada, y del $89 \%$ en la producción eléctrica obtenida. Estos datos demuestran el gran esfuerzo económico de este sector de producción eléctrica a pesar de las dificultades de distinta índole a las que ha tenido que hacer frente. 


\section{Cuadro 11}

Minicentrales hidroeléctricas, potencia instalada y producción eléctrica.

Años 1998 y 2013

\begin{tabular}{|l|c|c|c|c|c|c|}
\hline \multirow{2}{*}{$\begin{array}{c}\text { Comunidad } \\
\text { Autónoma }\end{array}$} & \multicolumn{2}{|c|}{ Centrales } & \multicolumn{2}{c|}{ Potencia MW } & \multicolumn{2}{c|}{ Producción GWh } \\
\cline { 2 - 7 } & $\mathbf{1 9 9 8}$ & $\mathbf{2 0 1 3}$ & $\mathbf{1 9 9 8}$ & $\mathbf{2 0 1 3}$ & $\mathbf{1 9 9 8}$ & $\mathbf{2 0 1 3}$ \\
\hline Andalucía & 23 & 32 & 75,05 & 118,40 & 200,66 & 267,44 \\
\hline Aragón & 29 & 55 & 96,08 & 187,53 & 401,04 & 697,40 \\
\hline Asturias & 10 & 17 & 27,14 & 36,66 & 54,91 & 100,19 \\
\hline Baleares & - & - & - & - & - & - \\
\hline Canarias & 1 & 1 & 0,46 & 046 & 1,34 & 3,05 \\
\hline Cantabria & 8 & 20 & 17,89 & 44,32 & 54,57 & 167,92 \\
\hline Castilla y León & 74 & 145 & 128,70 & 222,65 & 405,23 & 637,80 \\
\hline Castilla-La & 31 & 44 & 54,54 & 81,00 & 121,03 & 316,79 \\
Mancha & 96 & 144 & 168,41 & 212,23 & 569,66 & 827,92 \\
\hline Cataluña & 4 & 9 & 12,67 & 19,58 & 20,09 & 37,98 \\
\hline Extremadura & 45 & 84 & 105,08 & 302,10 & 276,74 & $1.140,79$ \\
\hline Galicia & 7 & 9 & 33,76 & 22,63 & 88,83 & 42,03 \\
\hline Madrid & 6 & 12 & 10,93 & 13,99 & 52,62 & 69,99 \\
\hline Murcia & 34 & 43 & 65,83 & 93,29 & 213,61 & 346,43 \\
\hline Navarra & 26 & 44 & 26,81 & 39,56 & 41,28 & 123,67 \\
\hline País Vasco & 13 & 18 & 19,44 & 27,00 & 67,25 & 94,61 \\
\hline La Rioja & 4 & 5 & 6,65 & 10,93 & 17,84 & 17,81 \\
\hline C. Valenciana & 411 & 682 & 849,44 & $1.477,87$ & $2.586,7$ & $4.891,82$ \\
\hline Total & & & & & & \\
\hline
\end{tabular}

Fuente: Comisión Nacional de Energía y Comisión Nacional de los Mercados y la Competencia.

\section{CONSIDERACIONES FINALES}

La energía hidroeléctrica ha sido en España y en el resto del Planeta pionera en la producción de electricidad limpia y próxima. La necesidad de abundante electricidad llevó a la construcción de grandes embalses, y al abandono de bastantes centrales de reducida potencia por su escasa rentabilidad.

La Política de Fomento de las Energías Renovables implantada en España desde finales del siglo pasado ha supuesto un importante proceso de reconversión para el sector de la energía minihidráulica, en su doble vertiente: recuperación de instalaciones abandonadas y construcción de nuevas centrales.

El análisis de los Planes de Fomento ha permitido conocer las directrices de esa Política para el fomento de esta tecnología, los obstáculos para su desarrollo 
y las propuestas de actuación de la Administración del Estado. Por tanto, corresponde a ella y al resto de administraciones demostrar si tienen interés para que continúe la aportación energética de esta fuente de energía autóctona y renovable.

\section{BIBLIOGRAFÍA}

Agencia Estatal de Evaluación de las Políticas Públicas y la Calidad de los Servicios (2011): Evaluación del Plan Español de Energías Renovables 20052010. Ministerio de Política Territorial y Administración Pública. Madrid, 249 pp.

ARRoYo ILERA, F. (2012): «El sistema eléctrico del Júcar y la electricidad madrileña», en Simposio Internacional Globalización, innovación y construcción de redes técnicas urbanas en América y Europa, 1890-1930. Brazilian Traction, Barcelona Traction y otros conglomerados financieros y técnicos. Barcelona, Universidad de Barcelona, Facultad de Geografía e Historia, 23-26 de enero 2012. pp. 1-23. Disponible: http:// www.ub.edu/geocrit/Simposio/cFArroyo_Elsistema.pdf

Espejo Marín, C. (2005): «La energía eléctrica en régimen especial en España», en Amica Verba in honorem Prof. Antonio Roldán Pérez. Universidad de Murcia. Murcia, pp. 249-263.

ESPEJO MARÍN, C. y GaRCíA MARÍN, R. (2010): «Agua y energía: producción hidroeléctrica en España», en Investigaciones Geográficas, n. ${ }^{\circ}$ 51, pp. 107-129.

European Small Hydropower Association (2006): Guía para el desarrollo de una pequeña central hidroeléctrica. Bruselas, $164 \mathrm{pp}$.

Instituto para la DiversificaCión y AHorro de la Energía (1999): Plan de Fomento de la Energías Renovables en España. Ministerio de Ciencia y Tecnología. Madrid, $271 \mathrm{pp}$.

Instituto para la DiversificaCión y Ahorro de la Energía (2005): Plan de Energías Renovables en España 2005-2010. Ministerio de Industria, Comercio y Turismo. Madrid, 350 pp.

Instituto PARA La DiversificaCión y Ahorro de la Energía (2011): Plan de Energías Renovables 2011-2020. Ministerio de Industria, Comercio y Turismo. Madrid, 774 pp.

Santos García, F. (2003): «Minicentrales hidráulicas», en De Juana, J.M. (Coord.): Energias renovables para el desarrollo. Paraninfo. Madrid, pp. 261-308.

UNESA (2003): «¿Qué es una minicentral hidroeléctrica?», en La electricidad en España. Madrid, pp. 77-78. 